\title{
PERFIL CLÍNICO E CRITÉRIOS DIAGNÓSTICOS DA MIELORRADICULOPATIA ESQUISTOSSOMÓTICA
}

\author{
Eustáquio Claret dos Santos', Gilberto Belisário Campos², Adriana Corrêa Diniz, \\ Juliana Cardoso Leaß, Manoel Otávio da Costa Rocha ${ }^{4}$
}

\begin{abstract}
RESUMO - Durante período de 20 anos (1972-1992), 56 pacientes com diagnóstico de mielorradiculopatia esquistossomótica foram internados em três hospitais de Belo Horizonte - Minas Gerais. Dados de cada paciente foram coletados retrospectivamente de seus prontuários. Em todos os casos, o diagnóstico foi presumido e baseou-se nos seguintes critérios: 1) quadro de comprometimento medular torácico baixo ou lombossacro (síndrome de cone medular e/ou cauda equina); 2) epidemiologia positiva para esquistossomose; 3) comprovação laboratorial de esquistossomose através de exame parasitológico de fezes ou biópsia retal; e 4) exclusão de outras patologias que pudessem causar quadro semelhante. Vários aspectos clínicos e epidemiológicos foram estudados para determinar o perfil diagnóstico da mielorradiculopatia esquistossomótica nesta amostra e são apresentados neste artigo. Reconhecendo os vários problemas no diagnóstico da mielorradiculopatia esquistossomótica, enfatizamos a importância de se pensar nesta entidade e sugerimos critérios para definição diagnóstica.
\end{abstract}

PALAVRAS-CHAVE: esquistossomose mansoni, radiculopatia, mielite.

Clinical profile and criteria for the diagnosis of schistosomotic myeloradiculopathy

\begin{abstract}
During 20 year period (1972-1992) 56 patients with a diagnosis of schistosomotic myeloradiculopathy were admitted in three hospitals of Belo Horizonte - Minas Gerais. Data from pacients were collected retrospectively from their medical records. In all cases, the diagnosis was inferred in a presumably way and was based on the following considerations: 1) the finding of low thoracic/upper lumbar neurological symptoms; 2) positive epidemiology for schistosomiasis; 3) demonstration of exposure to schistosomiasis through parasitologic or serologic techniques; and 4) the exclusion of other known causes of transverse myelitis and myeloradiculitis. Several clinical and epidemiological aspects were studied to determine the diagnosis profiles of the schistosomotic myeloradiculopathy in this sample and are presented in this paper. In recognizing the many problems in diagnosis of schistosomotic myeloradiculopathy we emphasize how important is thinking about this entity and criteria to improve diagnostic evaluation are suggested.
\end{abstract}

KEY WORDS: schistosomiasis, radiculopathy, myelitis.

A esquistossomose mansoni constitui uma das mais disseminadas parasitoses afetando o homem. Historicamente, corresponde a uma das mais velhas doenças conhecidas do mundo. O Egito foi, provavelmente, o primeiro foco do parasita, espalhando-se de lá para outras partes do continente africano e, mais tarde, para o Brasil, através do tráfico de escravos. Aqui, o parasita encontrou bom clima e hospedeiros favoráveis ${ }^{1}$. A doença constitui importante risco ocupacional em regiões tropicais e subtropicais ${ }^{2}$. No Brasil, representa um dos maiores problemas de saúde pública, estimando-se um total de 12 milhões de pessoas infectadas ${ }^{3}$.
O comprometimento do sistema nervoso no curso da esquistossomose mansoni vem ganhando, paulatinamente, importância cada vez maior na literatura especializada. As dificuldades no seu reconhecimento provavelmente limitam seu diagnóstico. Com relação ao sistema nervoso, não se conhece a frequência do seu envolvimento. Scrimgeour \& Gajdusek (1981) relatam que, na Tanzânia, a esquistossomose foi responsável por $1 \%$ dos casos de paraplegia não traumática e que, em $5 \%$ de outros casos, estabeleceu-se o seu diagnóstico presuntivo ${ }^{4}$. A neuroesquistossomose pode resultar numa grande variedade de complicações. Seu diagnóstico é difí-

Faculdade de Medicina da Universidade de Minas Gerais, Belo Horizonte MG, Brasil (FMUFMG): ${ }^{1}$ Médico Neurologista, Chefe do Serviço de Neurologia e Neurofisiologia; ${ }^{2}$ Professor Emérito de Neurologia; ${ }^{3}$ Acadêmica de Medicina; ${ }^{4}$ Professor Adjunto do Departamento de Clínica Médica. Apoio parcial: CNPq.

Recebido 6 Outubro 2000, recebido na forma final 10 Maio 2001. Aceito 16 Maio 2001. 
cil, podendo ser confundido com o de outras etiologias $^{5-8}$. Entretanto, a suspeita clínica da mielorradiculopatia esquistossomótica (MRE) não traz grandes dificuldades, apesar do diagnóstico, na maioria das vezes, ser presuntivo.

A inexistência de estudos que estabeleçam a freqüência da mielopatia na população esquistossomótica sugere a possibilidade que o diagnóstico, em muitos casos, passe despercebido, por falta de estudos sistemáticos visando estabelecer esta etiologia. Também, poderiam concorrer para a escassez de casos de MRE relatados, a baixa freqüência dos casos, a pouca consciência dos médicos, em nosso meio, para esta hipótese diagnóstica e a possibilidade de evolução frustra, oligossintomática ou transitória, com pouca perturbação funcional. Grande parte dos casos registrados na literatura, bem como os por nós catalogados ${ }^{7}$, receberam este diagnóstico de forma presuntiva. Os critérios usados são suficientes para o estabelecimento de um diagnóstico provável, mas não de certeza ${ }^{8}$.

É extensa a lista de possibilidades etiológicas a serem descartadas, o que complica a exequibilidade de propedêutica adequada em nosso meio, em virtude das dificuldades operacionais encontradas em nossas instituições de saúde ${ }^{9}$. Estes dados ilustram a precariedade da qualidade e apuro diagnóstico, reforçando a importância do estabelecimento de critérios mínimos necessários à formulação da hipótese de MRE.

\section{MÉTODO}

A presente pesquisa foi realizada através do levantamento de dados, de forma retrospectiva, em prontuários de pacientes com diagnóstico de mielorradiculopatia esquistossomótica, num período de 20 anos (1972 a 1992), em três hospitais da área metropolitana de Belo Horizonte, que são: Hospital das Clínicas da Universidade Federal de Minas Gerais (HC-UFMG), Hospital Felício Rocho (HFR), Hospital João XXIII (HJXXIII) da Fundação Hospitalar do Estado de Minas Gerais (FHEMIG). O método empregado consistiu na análise de prontuários de 56 pacientes com diagnóstico de mielorradiculopatia esquistossomótica, dos quais 31 estiveram internados no Serviço de Neurologia e Neurocirurgia do Hospital Felício Rocho, no período de julho de 1978 a março de 1991; 20 casos no Hospital das Clínicas da UFMG, no período de junho de 1978 a maio de 1992; e cinco casos no Hospital João XXIII da FHEMIG, no período de fevereiro de 1982 a julho de 1989.

Em todos os casos estudados, o diagnóstico de mielorradiculopatia esquistossomótica foi inferido clínica e laboratorialmente, sem comprovação histopatológica. Os critérios empregados no diagnóstico clínico e laboratorial foram os seguintes: 1) epidemiologia positiva para esquis- tossomose; 2) evidência clínica de lesão medular (torácica baixa, lombar ou sacral) e/ou de lesão de cone medular e cauda esquina; e, 3) presença de esquistossomose mansônica ativa, comprovada por exame parasitológico de fezes ou biópsia retal.

Em todos os casos analisou-se o hemograma, visando a identificação de eosinofilia, exame parasitológico de fezes (EPF) e/ou biópsia retal, reação imunológica para sífilis no sangue e líquor, e a reação de fixação do complemento para cisticercose no líquor. Através dos dados de anamnese e exame clínico, foram afastadas as possibilidades diagnósticas de doenças hereditárias (paraparesia espástica hereditária), poliomielite, esclerose múltipla, esclerose lateral amiotrófica, polirradiculoneurites, degeneração combinada de medula, condições patológicas de natureza tóxica (benzol, arsênico, mercúrio), física (pós-radiação), ou secundária ao uso de medicamentos (clioquinol, sulfas, penicilina, anestésicos).

Os dados analisados compreenderam a anamnese, o exame neurológico, os exames complementares realizados, o tipo de tratamento empregado e a evolução clínica. Foram também avaliadas as dificuldades encontradas na apuração dos dados, devido a falhas na descrição dos exames neurológicos e complementares. Em relação à anamnese, procurou-se atentar para o tempo decorrido entre o início das manifestações clínicas e o alcance à atenção médica. Os sinais e sintomas (dor, fraqueza muscular, parestesia, retenção urinária) foram tabulados de forma a se analisar sua frequência de forma isolada, ou em associação. Os principais achados descritos nos exames neurológicos foram sistematizados de forma a permitir a avaliação de sua frequência.

Os exames laboratoriais analisados consistiram do exame parasitológico de fezes, da biópsia retal, e do estudo do líquor. Em poucos casos foi realizada mielografia. Em relação ao estudo do líquor, foram analisados a citometria global, a predominância citológica, a pesquisa de eosinófilos, o teor de proteínas e de glicose.

Com relação ao tratamento, foram analisados seu emprego em relação ao tempo de instalação do quadro clínico, o tipo de tratamento realizado e a correlação com a evolução clínica.

\section{RESULTADOS}

Dos 56 pacientes levantados, houve predominância do sexo masculino numa proporção de 4:1. A distribuição segundo a faixa etária mostrou predominância de casos na idade entre 20 a 29 anos. A menor idade encontrada foi de três anos e, a maior, de 56 , com média de $28,86 \pm 12,43$ anos. Os dados relativos à cor evidenciam predomínio, dos leucodérmicos, seguido dos feodermas, e por último, dos melanodérmicos. A maioria dos pacientes era proveniente da área metropolitana de Belo Horizonte, Minas Gerais, $(69,6 \%)$, e apenas um paciente procedia de outro estado $(1,8 \%)$. 
Em relação à anamnese e ao exame neurológico, a queixa de retenção urinária ocorreu em 42 pacientes $(75,0 \%)$, dor esteve presente em $43(76,8 \%)$, parestesia em 41 (73,2\%), fraqueza em $48(85,7 \%)$, sendo esta a queixa mais comum. Dos 56 pacientes, os reflexos patelar e aquileu foram analisados em 51 e 50 pacientes, respectivamente. Observou-se que o reflexo patelar estava normal em 13 pacientes, abolido em 21, diminuído em cinco e aumentado em 12. O reflexo aquileu estava anormal em dez pacientes, abolido em 27, diminuído em três e aumentado em dez. Quanto ao nível sensitivo, o maior acometimento ocorreu, em ordem de freqüência, nos segmentos lombares altos e torácicos baixos. O nível mais alto encontrado foi T4 e o mais baixo $\mathrm{S} 1$.

O tempo decorrido entre a instalação dos sintomas e a admissão hospitalar foi extremamente variável. A média geral encontrada foi de 52,6 dias, com variação de um a 900 dias. A média de tempo de permanência hospitalar variou de quatro a 108 dias, com média de 22,02 $\pm 19,56$ dias.

Os critérios observados na distinção entre as formas clínicas obedeceu, puramente, à presença de dores radiculares, déficit sensitivo, distúrbio esfinctérico e alterações de reflexos, e foram classificados de acordo com BIRD $^{10}$. O maior número de casos correspondeu à forma mielorradicular, com $31 \mathrm{ca}-$ sos $(55,3 \%)$, seguido pela forma mielítica, com 15 casos $(28,6 \%)$. Verificou-se um caso com a forma pseudotumoral (1,8\%). Em nove pacientes $(16,1 \%)$ não foi possível estabelecer a forma clínica.

Do ponto de vista laboratorial, o exame parasitológico de fezes (EPF) foi positivo em $77,4 \%$ dos 31 casos em que este exame foi realizado. A biópsia retal, realizada em 45 pacientes, evidenciou presença de ovos de $S$. mansoni em todos eles (100\%). A positividade de ambos os métodos, realizados simultaneamente em 18 casos, foi encontrada em 11.

0 estudo do líquor foi realizado em 55 pacientes. O número de células estava normal em $21,4 \%$ dos casos. Predominou a pleocitose moderada, até 200 células $/ \mathrm{mm}^{3}$, presente em 38 casos $(69,1 \%)$. 0 número máximo de células encontradas foi de 699 células/ $\mathrm{mm}^{3}$. Em $98 \%$ dos casos, houve predomínio de mononucleares. Apenas em um caso ( $2 \%$ ) observou-se predomínio de polimorfonucleares. O conteúdo de proteínas do líquido cefalorraquidiano estava alterado em 37 casos (72,5\%) e normal em 14 $(27,5 \%)$. O teor de glicose no líquor não mostrou alteração. A eosinofilorraquia foi encontrada em 32 casos $(60,4 \%)$, e eosinofilia no sangue foi observado em $11(20,4 \%)$.
A mielografia foi realizada em 20 casos. Destes, apenas um mostrou sinais de bloqueio com nível em L1 - L2. O paciente foi submetido à laminectomia, sendo encontrado espessamento da leptomeninge, com presença de pontos branquicentos na superfície medular e ingurgitamento vascular. $O$ estudo anatomopatológico não detectou ovos ou granulomas.

Em poucos casos foi possível determinar a forma clínica da parasitose. Houve predomínio da forma intestinal, encontrada em dez casos $(17,9 \%)$. Verificou-se um caso, apenas, para as demais formas (agu$\mathrm{da}$, hepatoesplênica e hepatointestinal). Em 43 relatos $(76,8 \%)$ este dado não estava disponível nos prontuários.

Os pacientes foram tratados de forma variada, utilizando-se terapêutica específica isolada ou associada à corticoterapia. Esta última também foi usada em alguns casos, isoladamente. A maioria dos pacientes recebeu a associação de prednisona com oxamniquina ( 32 casos $/ 57,1 \%$ ). Prednisona foi administrada isoladamente em 12 casos $(21,4 \%)$ e dexametasona, associada à oxamniquina, em três casos $(5,4 \%)$. O praziquantel foi usado em seis pacientes $(10,7 \%)$ concomitantemente à prednisona. Apenas um paciente $(1,8 \%)$ usou oxamniquina isoladamente e, em dois casos (3,58\%), nenhum tratamento foi instituído.

Em sete casos, não houve informação quanto ao quadro evolutivo. Nos demais, foi observada melhora em $62,5 \%$ (35 casos). Em treze pacientes (23,2\%) não houve modificação do quadro clínico. Um paciente $(1,8 \%)$ apresentou piora.

\section{DISCUSSÃO}

O diagnóstico de certeza da mielorradiculopatia esquistossomótica repousa na confirmação anatomopatológica, seja por biópsia ou achado post-mortem. A maioria dos casos publicados na literatura, sem confirmação histológica, também não recebeu exploração extensiva de outros diagnósticos diferenciais. Além disso, a melhora clínica com o tratamento específico foi considerada, por alguns autores, como confirmação diagnóstica ${ }^{11-15}$.

Em relação ao estabelecimento da freqüência da mielorradiculopatia esquistossomótica, poucos são os trabalhos que se referem a esta questão. Basicamente, encontram-se dados obtidos por Scrimgeour, que relatou incidência de $6 \%$ de mielopatia atribuída à esquistossomose, numa série de 100 pacientes com paraplegia não-traumática ${ }^{4}$. Spina-França et al. relataram incidência de $5,6 \%$ de mielopatia, numa 
série de 353 pacientes com mielopatias não-traumáticas e não-tumorais, internados no Hospital das Clínicas da Faculdade de Medicina da Universidade de São Paulo (HC-FMSP) ${ }^{16}$. Galvão obteve freqüência de $0,3 \%$ de casos, num total de 9765 pacientes, internados na Clínica Neurológica do HC-FMSP, num período de 17 anos $^{17}$.

Apuramos na literatura, nacional e estrangeira, um total de 181 casos descritos ${ }^{7}$. Destes, o diagnóstico foi feito através de análise histopatológica em 57 casos $(31,3 \%)$, sendo $37(20,6 \%)$ à biópsia de material cirúrgico, e 20 (11,1\%), através de achado de necrópsia.

Com relação ao sexo, observa-se, na literatura consultada, nítida predominância do sexo masculino em relação ao feminino ${ }^{5,10,14,18}$. Em nossa série, observou-se proporção de 3,6:1 (78,6\% masculino e $23,4 \%$ feminino). Este fato pode refletir a maior probabilidade de contágio em pessoas do sexo masculino, devido a fatores sócio-econômicos (tipo de trabalho, banho em rios e lagoas), assim como ao maior esforço físico por eles dispendido, que aumentaria a pressão intra-abdominal ${ }^{14}$.

A terceira década respondeu pela maior taxa de distribuição dos casos $(41,1 \%)$, em conformidade com os dados catalogados na literatura $(32,6 \%)$. É possível que também esta proporção possa ser devida a fatores sócio-econômicos.

Dos sintomas observados, a dor de localização lombar e sacral foi encontrada em 76,8\% (43 pacientes), respondendo pelo sintoma inicial. $\mathrm{Na}$ série de Galvão, a dor constitui sintoma prodrômico, sendo encontrada em $75 \%$ dos casos analisados ${ }^{9}$. Nos casos relatados por Costa et al., a lombociatalgia esteve presente em $72,2 \%$ dos pacientes ${ }^{15}$. Nas casuísticas de Peregrino et al. e Ferrari et al., esta manifestação ocorreu em 100 e $96 \%$ dos casos, respectivamente ${ }^{14,19}$.

Retenção urinária e parestesia em membros inferiores vieram a seguir, com $75 \%$ e $73,2 \%$, respectivamente. Incontinência urinária esteve presente em sete pacientes $(12,5 \%)$. Estes sintomas são bastantes característicos da doença, de acordo com a literatura consultada $14,15,17,20,21$.

Em relação ao exame neurológico, fraqueza nos membros inferiores esteve presente em $85 \%$ dos casos. A paraparesia foi bilateral e simétrica em todos os casos observados. Este achado coincide com os relatos das principais séries analisadas $14,15,17,19$. Os reflexos profundos mostraram-se abolidos ou diminuídos na proporção de $51 \%$, para o patelar, e $60 \%$, para o aquileu. Hiperreflexia foi observada em $20 \%$ dos casos.
As lesões medulares na esquistossomose se distribuem preferencialmente nos segmentos inferiorestorácicos baixos e lombossacros, com características de síndrome de cone medular e cauda equina ${ }^{14,17,22}$, o que vai ao encontro de nossos achados $(86,4 \%)$.

Problemas relacionados à potência sexual foram registrados em apenas três casos $(5,4 \%)$, discordando de outras casuísticas, como as de Galvão e a de Peregrino et al., que mostraram comprometimento em $100 \%$ dos $\operatorname{casos}^{14,17}$. Esta cifra baixa encontrada pode se dever à falta da investigação sistemática deste dado na anamnese e também à não-informação espontânea por parte do paciente.

A predominância da forma mielorradicular (71 casos/44,4\%), seguida pela mielítica (44 casos/ $27,5 \%$ ) e pela pseudotumoral (39 casos $/ 24,4 \%$ ) não difere dos achados encontrados na literatura.

Dos exames laboratoriais, o parasitológico de fezes apresenta positividade variável nas grandes séries publicadas (38,4\% na série de Galvão; $42,8 \%$ na de Peregrino et al.; e 59,5\% na Costa et al.). Verificamos $77,4 \%$ de exames positivos. Na imensa maioria dos casos estudados, apenas se realizou um exame parasitológico de fezes.

Com relação à biópsia retal, o encontro de ovos de $S$. mansoni tem variado de $47,6 \%^{14}$ a $69,2 \%{ }^{17}$ e $83,3 \%^{15}$. Verificou-se $100 \%$ de positividade nos 45 pacientes submetidos ao exame nesta série. Rabello verificou que a biópsia retal apresenta maior sensibilidade quando comparada ao exame de uma única amostra fecal, pelos métodos de Lutz/Hoffman, Pons \& Janer ou Kato-Katz, e igual sensibilidade quando comparada a duas amostras ${ }^{23}$.

O exame do líquor estava normal em seis casos $(10,7 \%)$. Pleocitose até 200 leucócitos por $\mathrm{mm}^{3}$ foi observada em 38 casos $(69,1 \%)$ e acima deste valor em cinco casos $(9,1 \%)$. O predomínio de linfomononucleares alcançou percentual de 98,2\%. Em apenas um caso houve predomínio de polimorfonucleares. O teor de proteínas estava normal em 27,5\% e elevado em $72,5 \%$. Na grande maioria dos casos, as taxas ficaram entre 51 e $200 \mathrm{mg} \%(67,2 \%)$. O valor máximo encontrado foi de $2,8 \mathrm{~g} \%$. 0 encontro de eosinofilorraquia ocorreu em $60,4 \%$. Os achados liquóricos encontrados coincidem com o padrão descrito por Livramento et al., caracterizado por pleocitose às custas de linfomononucleares, hiperproteinorraquia, presença de células eosinófilas, e aumento do teor de globulinas gama ${ }^{24}$. Este último sem informações nos exames estudados. Testes imunológicos não foram realizados nos pacientes aqui 
Quadro 1. Critérios para o diagnóstico da mielorradiculopatia esquistossomótica.

\begin{tabular}{|c|c|}
\hline \multirow[t]{5}{*}{ Diagnóstico Comprovado } & Comprometimento medular torácico baixo ou lombossacro; \\
\hline & Epidemiologia positiva para esquistossomose mansônica; \\
\hline & Exame parasitológico de fezes e/ou biópsia retal positiva para S. mansoni; \\
\hline & Exclusão de outras patologias; \\
\hline & Biópsia de material cirúrgico com ovos $S$. mansoni. \\
\hline \multirow[t]{4}{*}{ Diagnóstico provável } & Comprometimento medular torácico baixo ou lombossacro; \\
\hline & Epidemiologia positiva para esquistossomose mansônica; \\
\hline & Exame parasitológico de fezes e/ou biópsia retal positiva para S. mansoni; \\
\hline & Exclusão de outras patologias. \\
\hline \multirow[t]{2}{*}{ Diagnóstico possível } & Comprometimento medular torácico baixo ou lombossacro; \\
\hline & Epidemiologia positiva para esquistossomose mansônica. \\
\hline
\end{tabular}

Observação: 0 estudo do líquor, que deve ser realizado de rotina, pode apresentar alterações ${ }^{18,20,24,26,27}$ que, para alguns autores, sugerem a etiologia esquistossomótica, tais como pleocitose linfomononuclear, aumento de proteína, eosinofilorraquia e, hipergamaglobulinorraquia ${ }^{24}$. Com relação à reatividade de reações imunológicas específicas para esquistossomose (imunofluorescência, ELISA, hemoaglutinação e/ou Western Blot), são poucos os estudos realizados, com casuística escassa, rescindindo-se, ainda, de padronização e normatização de valores normais e patológicos ${ }^{14,17,24,28,29}$. Com a padronização destes testes, estes critérios poderão sofrer modificações.

catalogados. Na casuística de Galvão, o teste com a esquistossomina foi positivo em $80 \%$ dos casos, a imunofluorescência em $77,7 \%$, e as reações de fixação do complemento e hemaglutinação em $100 \%$ dos pacientes testados ${ }^{17}$.

Apenas três pacientes foram submetidos a exame eletroneuromiográfico, que revelou sinais de desnervação em todos, caracterizando o comprometimento radicular.

Em relação à forma clínica da esquistossomose, observou-se um caso com forma aguda, dez com a forma intestinal, um com a forma hepatoesplênica, um com forma hepatointestinal e 43 casos não determinados. Nenhum caso apresentou quadro clínico compatível com forma pulmonar.

A corticoterapia foi usada em $94,6 \%$ dos casos, associada a tratamento específico em $73,2 \%$ e isoladamente em $21,4 \%$. Os corticóides usados foram a prednisona $(89,2 \%)$ e a dexametasona $(5,4 \%)$. Não houve padronização das doses. Os pacientes receberam alta hospitalar, em uso de corticóide, não sendo possível determinar o tempo de duração da droga. Apenas um caso foi tratado exclusivamente com oxamniquina. Em um caso não foi realizado terapêutica e, em outro, não houve dados disponíveis.

Em 35 casos, houve melhora do quadro. Treze pacientes permaneceram inalterados, um piorou e, em sete casos, não se dispôs de dados sobre a evolução. A melhor resposta foi observada no grupo que fez uso da associação de corticóide com tratamento específico.

Devido à disparidade entre os grupos, não se pode tecer comentários quanto ao melhor tipo de corticóide ou de tratamento específico. O paciente que mostrou piora corresponde ao que foi submetido à laminectomia e uso de corticoterapia isoladamente. Este fato reforça os dados da literatura, os pacientes que sofreram intervenção cirúrgica apresentaram índices de melhora mais baixos, independentemente de terem ou não recebido tratamento específico e/ou corticóide após a cirurgia ${ }^{17}$.

Neste estudo, a média de tempo decorrido entre o início dos sintomas e a admissão hospitalar foi de 52,6 dias, com mínimo de um e máximo de 900 dias. Houve concordância com os dados publicados de 56,42 dias (desvio padrão de 121,97), com mínimo de dois e máximo de 730 dias $^{17}$. 0 tempo de aparecimento da complicação neurológica, em relação ao início da infecção, não pôde ser inferido, devido à impossibilidade de se precisar a data provável do contágio. A variação observada nas médias obtidas entre o início dos sintomas e a admissão hospitalar reflete, por um lado, as dificuldades de acesso dos pacientes ao nível de atenção terciária e, ultrapassada esta barreira, a fatores relacionados ao número de leitos disponíveis para internação, geralmente reduzidos.

Alguns autores têm proposto o uso de protocolos com fins de padronização propedêutica, aumentando a possibilidade diagnóstica e permitindo o tratamento precoce ${ }^{14,25}$. É de grande importância o planejamento e aplicação destes protocolos objetivando diagnóstico e tratamento precoces, evitando o surgimento de quadros incapacitantes.

Em virtude da necessidade de se aventar este diagnóstico, e em decorrência das dificuldades de se realizar a propedêutica de forma rápida, sugerimos cri- 
térios para o diagnóstico clínico da mielorradiculopatia esquistossomótica. Este fato se prende às seguintes razões: 1 . necessidade de se levantar esta hipótese para qualquer paciente, em nosso meio, com sinais sugestivos de comprometimento medular não-traumático; 2 . necessidade de coleta de dados epidemiológicos, clínicos e laboratoriais que embasem o diagnóstico em diversos níveis de certeza, de preferência mais elevada; e, 3. necessidade de um mínimo de embasamento diagnóstico, para que o paciente seja submetido a tratamento precoce. O Quadro 1 apresenta a proposta de critérios para o diagnóstico de MRE, por nós sugerida.

\section{REFERÊNCIAS}

1. Jabur P. Novo caso de esquistossomose mansônica autóctone do estado de São Paulo: síndrome de cauda eqüina de provável etiologia esquistossomótica. Arq Hosp Santa Casa São Paulo 1960; 6:75-82.

2. Hatz C, Savioli L, Mayombana C, Dhunputh J, Kisumku UM, Tanner M. Measurement of schistosomiasis-related morbidity at community level in areas of different endemicity. Bull WHO 1990; 68: 777-787.

3. Lambertucci JR, Rocha RS, Carvalho OS, Katz N. A Esquistossomose mansoni em Minas Gerais. Rev Soc Bras Med Trop 1987;20:47-52.

4. Scrimgeour EM. Non-traumatic paraplegia in northerrn Tanzania. Brit Med J 1981;283:975-978.

5. Scrimgeour EM, Gajdusek DC. Involvement of the central nervous system in Schistosoma mansoni and S. haematobium infection: a review. Brain 1985;108:1023-1038.

6. Haribhai HC, Bhigjel AI, Bill PLA, et al. Spinal cord schistosomiasis. Brain 1991;114:709-726.

7. Santos EC. Perfil diagnóstico da mielorradiculopatia esquistossomótica em três hospitais de Belo Horizonte - Minas Gerais. Dissertação de Mestrado, Faculdade de Medicina da Universidade Federal de Minas Gerais. Belo Horizonte, 1994.

8. Bia FJ, Barry, M. Parasitic infections of the central nervous system. Neurol Clin North Amer 1986;4:171-206.

9. Peregrino, AJP. Neuroesquistossomose. In Machado LR, Livramento JA, Nóbrega JPS, Gomes HR, Spina-França A (eds). Neuroinfecção 98. São Paulo: Academia Brasileira de Neurologia, 1998:45-50.

10. Bird AV. Acute spinal schistosomiasis. Neurology 1964;14:647-656.
11. Couto D, Couto B. Schistossomiasis of the spinal cord. Rev Neurol 1962;20:315-325.

12. Molyneux ME, Galatius-Jensen F. Successful drug treatment of schistosomal myelopathy; a case report. S Afr Med J 1978;54:871-872.

13. Scaff M, Riva D, Spina-França A. Meningorradiculomielopatia esquistossomótica. Arq Neuropsiquiatr 1971;29:227-233.

14. Peregrino AJP, Oliveira SP, Porto CA, et al. Meningomielorradiculite por Schistossoma mansoni. Arq Neuropsiquiatr 1988;46:49-60.

15. Costa RO., Gameleira FT, Tenorio RB, Bras LH, Costa VB, Pinto Jr JM Neuroesquistossomose em Alagoas. Rev Bras Neurol 1992;28:79-84.

16. Spina-França A, Salum PNB, Limongi JCP, Berger A, Losso ER Mielopatias: aspectos diagnósticos. Arq Neuropsiquiatr 1980;38:360-366.

17. Galvão ACR. Mielopatias esquistossomóticas; aspectos clínicos e laboratoriais. Dissertação de Mestrado: Faculdade de Medicina da Universidade de São Paulo. São Paulo, 1983.

18. Salum PNB, Machado LR, Spina-França A. Meningomielorradiculopatia na esquistossomose mansônica; avaliação clínica e do líquido cefalorraqueano em 16 casos. Arq Neuropsiquiatr 1981;39:289-295.

19. Ferrari TCA, Moreira PRR, Ferrari MLA., et al. Clinical and immunological study of schistosomal myelorradiculopathy. Ann Trop Med Parasitol 1993;87:295-297.

20. Rocha FJ, Barros JA, Fonseca AVB, Ferreira ROMF. Esquistossomose da medula espinal. Rev Assoc Med Minas Gerais 1977;28:68-71.

21. Lacoste D, Delmas M, Longy M, Derrien JD, Le Bras M, Moretti G. Les myelopathies bilharziennes. Med Trop 1980;40:295-300.

22. Houpis J, Oexmann J, Martin J, Jacobi G, Reardon J, Waterman G. Acute schistosomiasis with tranverse myelitis in american students returning from Kenya. M M W R 1984;33:445-447.

23. Rabello ALT O exame parasitológico de fezes, a biópsia retal e o ensaio-imunoenzimático no diagnóstico da esquistossomose mansoni humana. Dissertação de Mestrado, Faculdade de Medicina da Universidade Federal de Minas Gerais. Belo Horizonte, 1990.

24. Livramento JA, Machado LR, Caetano da Silva L, Spina-França A. Síndrome do líquido cefalorraquidiano na neuroesquistossomose. Arq Neuropsiquiatr 1985;43:372-377.

25. Haribhai HC, Bhigjee AI, Bill PLA, et al. Spinal cord schistosomiasis. Brain 1991;114:709-726.

26. Cohen J Capildeo R, Clifford Rose F, Pallis C. Schistosomal myelopathy. Br Med J 1977;1:1258.

27. Herskowitz A. Spinal cord involvement with Schistosoma mansoni. J Neurosurgery 1972;2:494-498.

28. Pammenter MD, Haribhai HC, Epstein SR, Rossouw EJ, Bhigjee AI, Bill PLA. The value of immunological approaches to the diagnosis of Schistosomal myelopathy. Am J Trop Med Hyg 1991;44:329-335.

29. Ferrari TCA. Spinal Cord Schistosomiasis. A report of 2 cases and review emphasizing clinical aspects. Medicine 1999:78,176-190. 